\title{
11 Experimental In Vivo Models of Multiple Sclerosis: State of the Art
}

\author{
SARA PALUMBO ${ }^{1} \bullet$ SILVIA PELLEGRINI²
}

${ }^{1}$ Department of Surgical, Medical, Molecular Pathology and Critical Care, University of Pisa, Pisa, Italy; ${ }^{2}$ Department of Experimental and Clinical Medicine, University of Pisa, Pisa, Italy

Author for correspondence: Sara Palumbo, Department of Surgical, Medical, Molecular Pathology and Critical Care, University of Pisa, via Savi 10, I-56126 Pisa, Italy. E-mail: sara.palumbo@for.unipi.it Doi: http://dx.doi.org/10.15586/codon.multiplesclerosis.2017.ch11

\begin{abstract}
Multiple sclerosis is a multifactorial and heterogeneous neurological disease; hence, several experimental animal models had to be developed to mimic the different features of human pathology. Three main classes of animal models have been developed:experimental autoimmune encephalomyelitis (EAE), cuprizone intoxication, and Theiler's murine encephalomyelitis virus (TMEV) infection. The EAE model is the most versatile as it allows the reproduction of different patterns of multiple sclerosis; it is mostly relevant for relapsing-remitting multiple sclerosis and has allowed the development of several first-line, disease-modifying drugs for the treatment of multiple sclerosis. The other two models are less flexible than the EAE model and, to date, have not led to the discovery of any clinically relevant therapies. The cuprizone model mostly mimics the acute and chronic courses of multiple sclerosis, and it may represent a useful tool to develop novel therapies to protect oligodendrocytes and stimulate remyelination. Finally, the TMEV infection is the reference model to specifically study viral-mediated mechanisms of acute and primary progressive multiple sclerosis.
\end{abstract}

In: Multiple Sclerosis: Perspectives in Treatment and Pathogenesis. Ian S. Zagon and Patricia J. McLaughlin (Editors), Codon Publications, Brisbane, Australia. ISBN: 978-0-9944381-3-3; Doi: http://dx.doi.org/10.15586/codon.multiplesclerosis.2017

Copyright: The Authors.

Licence: This open access article is licenced under Creative Commons Attribution 4.0 International (CC BY-NC 4.0). https://creativecommons.org/licenses/by-nc/4.0/ 
Key words: Cuprizone; EAE; In vivo models; Multiple sclerosis; TMEV

\section{Introduction}

Multiple sclerosis is a complex and heterogeneous neurological illness with regard to its pathological phenotype (e.g., primary progressive, secondary progressive, and relapsing-remitting) (1) and etiology (e.g., autoimmune-dependent and autoimmune-independent) $(2,3)$. Although many conflicting hypotheses exist about the nature of the primary hit triggering this pathology (e.g., multiple genetic predisposing factors in interaction with different environmental factors) (4), multiple sclerosis is characterized by the concomitant manifestation of a wide range of specific biological alterations. For instance, demyelination, inflammation, astrogliosis, microglia activation, macrophage and lymphocyte infiltration, and axonal damage represent common hallmarks of this pathology (5-8). Due to the large number of molecular mechanisms, variability of this disease among patients, and uncertain etiology, the following three experimental animal models, each reproducing different features of human pathology, have been developed: the experimental autoimmune encephalomyelitis (EAE) model, the cuprizone intoxication model, and the Theiler's murine encephalomyelitis virus infection (TMEV) model. In this chapter, the characteristics of these animal models, the procedures of induction, the main biological features, and their relevance in multiple sclerosis research are described.

\section{The EAE Model of Multiple Sclerosis}

Since 1947, when Walt and colleagues suggested that the EAE is a suitable experimental model for multiple sclerosis, many research projects have employed this model to investigate the pathophysiological mechanisms underlying human multiple sclerosis and to test new therapies (9). EAE is characterized by an autoimmune reaction against the myelin proteins in the central nervous system. Two distinct protocols are used to induce EAE, the administration of activated T-lymphocytes that act specifically against myelin antigens or, more frequently, the administration of myelin-derived peptides, which, in turn, cause an immune reaction against specific antigenic myelin proteins. Different types of peptides, such as the myelin basic protein (MBP), proteolipid protein (PLP), myelin oligodendrocyte glycoprotein (MOG), and several of their encephalitogenic epitopes are used to induce EAE (10). The peptides are generally administered via subcutaneous injection, solubilized in complete Freund's adjuvant solution, which functions as a depot of antigens for a prolonged and continuous release of the active peptides. However, in 2002, it was pointed out that this adjuvant exerts some inhibitory activities on EAE pathology, suggesting that it should be used with caution (11). More recently, it has been shown that EAE can be induced even without the Freund's adjuvant (12).

Three lymphocytic cell populations mediate the induction of EAE, Thl, and Th17 types of the $\mathrm{CD}^{+}$cells, and $\mathrm{CD} 8^{+}$T-lymphocytes, with the CD4+ lymphocytes being the main mediators; after entering the central nervous system, these 
cells target myelin proteins and mature oligodendrocytes causing myelin degradation, axonal damage, and oligodendrocyte apoptosis (13-16). The addition of the pertussis toxin to the injection mixture facilitates the migration of the lymphocytes across the blood-brain barrier (17). The migration of T-cells into the brain is typically accompanied by monocyte and/or macrophage infiltration and activation (18). Moreover, resident microglia and astrocytes actively respond to the insult and undergo activation as well. All these cell types have been shown to produce and release inflammatory mediators, such as chemokines and cytokines, thus contributing to the axonal damage and demyelination $(18,19)$.

In the EAE model, the peak of demyelination is reached after 10-15 days from the injection, primarily confined to the spinal cord, although a certain degree of demyelination is also detected in the optic nerve, cerebral cortex, and cerebellum $(20,21)$. Moreover, axonal damage and generalized paralysis are progressively developed with demyelination (8). Specifically, the paralysis starts from the tail, then affects the hind limbs, and ultimately compromises the forelimbs.

The pathological characteristics of EAE are not uniform as they considerably vary depending on the type of the epitope and the type of the animal used. For instance, in C57BL/6 mice, encephalitogenic epitopes of MOG induce a chronic progressive disease, whereas in NOD/Lt and SJL mice and Lewis rats they cause a chronic relapsing-remitting disease with variable severity $(17,22,23)$. Susceptibility to EAE is modulated by genetic factors that influence the response to myelin antigens. For instance, B6 and SJL mice are resistant to MBP immunization, but they respond well to MOG treatment. This variability seems to be modulated by some polymorphic regions within the major histocompatibility complex genes (24-25). In PL/J mice, the epitope injection induces a noncanonical form of relapsing-remitting disease (26). Interestingly, in SJL mice, a spontaneous relapsing-remitting EAE can be induced if the mice have been previously engineered to carry a specific T-cell receptor for myelin oligodendrocyte glycoprotein (27). Finally, the disease course differs between genders; for example, SJL, ASW, and NZW females show a higher incidence of EAE, resembling the higher prevalence of multiple sclerosis in women when compared to men (28).

Lewis is the most commonly used rat strain for EAE. Lewis rats develop brain pathology without the need of pertussis toxin that represents an artifact with regard to human pathology. However, inducing EAE in Lewis rats presents several drawbacks, as the obtained pathological phenotype lacks fundamental hallmarks of human multiple sclerosis. In particular, different to the human pathology, demyelination is not clearly detected and inflammation is not widespread in the whole brain, but mostly localized in the spinal cord. Even though rats have been considered valid experimental animals to study the activity of the immune cells in the central nervous system, they have been gradually supplanted by mice for multiple sclerosis research. Mice are easier to handle and particularly convenient for genetic manipulation (29). In addition to mice and rats, EAE can be induced in many other animal species like primates, rabbits, and guinea pigs (30-33). In summary, EAE reproduces many aspects of multiple sclerosis in terms of disease course, pathogenic mechanisms, and pathological features. In particular, myelin degradation and axonal damage are prominent in the spinal cord, consequent to autoimmune processes primarily mediated by the infiltrating $\mathrm{CD} 4^{+} \mathrm{T}$-lymphocytes. EAE is broadly deemed to be a good model to test immunosuppressive therapeutic agents, as demonstrated by the fact that it led to the establishment of several clinically relevant therapies $(34,35)$. 


\section{The Cuprizone Model of Multiple Sclerosis}

The intoxication models of demyelination are based on the administration to laboratory animals of bioactive molecules that specifically target oligodendrocytes causing their degeneration and death, ultimately leading to severe demyelination in the brain. Several toxins such as ethidium bromide, lysolecithin, and cuprizone have been shown to efficiently trigger demyelination in the central nervous system (36). Of these, cuprizone is widely used in multiple sclerosis research. Cuprizone, bis-cyclohexanone oxaldihydrazone, is a neurotoxic copper chelator agent. Its deleterious effects on rodent brain were discovered by the pioneering work of Carlton in 1966 (37). Administered in the past, in addition to Swiss, CD1, and ICI mice (38), to other species, like guinea pigs, today cuprizone is prevalently used in mice $(37,39)$. It has been suggested that rats do not develop demyelination with cuprizone as consistently and reproducibly as mice do and that several rat brain areas remain completely unaffected (40). However, recent studies show that Wistar rats, in response to cuprizone, develop widespread demyelination of the cortex, corpus callosum, and cerebellum $(41,42)$ suggesting that rats, similarly to mice, are suitable for longitudinal studies. Indeed, rats could be a better choice for imaging studies due to their larger size (42).

C57BL/6 is the most widely used strain of mice for the induction of the cuprizone-mediated multiple sclerosis. In this strain, a minimal dosage of the compound is sufficient to cause highly reproducible brain pathology with limited peripheral side effects, such as weight loss and liver toxicity. As established by Hiremat and colleagues in 1998, cuprizone is administered per os by using a $0.2 \% \mathrm{w} / \mathrm{w}$ powdered rodent standard chow ad libitum for 5-6 weeks to C57BL/6 mice aged 8-10 weeks (43). After 6 weeks of cuprizone diet, a maximum of demyelination is reached within the gray and white matter, especially in the corpus callosum area (43) and the superior cerebellar peduncles $(44,45)$, but not in the spinal cord (46); motor disabilities become prominent (43). The demyelination process is characterized by selective and progressive apoptosis of mature oligodendrocytes, axonal pathology, activation of astrocytes and microglia, infiltration of macrophages and inflammation (43-45, 47-49). The inflammatory burden is characterized by the production of cytokines, interleukins, tumor necrosis factor, and arachidonic acid metabolizing enzyme, and by the consequent production of lipoxins, thromboxane, and proinflammatory prostaglandins that play an active role in the severity of demyelination $(47,48,50,51)$. An intact blood-brain barrier with no signs of lymphocyte infiltration have been observed in the cuprizone model $(52,53)$.

The interruption of cuprizone feeding after 6 weeks of continuous intoxication, immediately after peak demyelination has reached, allows for a spontaneous remyelination of the brain and a complete recovery in a time lapse of six additional weeks (47). For this reason, the cuprizone model is also used to investigate the mechanisms of remyelination. Prolonged administration of cuprizone, for 6-7 months, impairs remyelination as in progressive multiple sclerosis (54). Cuprizone can also be administered in repeated doses mimicking the course of relapsingremitting multiple sclerosis (55). In summary, cuprizone allows an experimental reproduction of different pathological courses, such as the acute, chronic, and relapsing-remitting forms of multiple sclerosis. 
Given these characteristics, the cuprizone model allows investigators to selectively study demyelination and remyelination processes, independently from the effects of the immune system. It is mostly used to test new pharmacological treatments to counteract demyelination and to favor remyelination. Remyelination, in fact, can be severely impaired in multiple sclerosis, because of dysfunctional and inefficient maturation of oligodendrocyte precursors. However, the recommended pharmacological therapies, currently used in clinics, have no specific activity on remyelination; thus, the need to develop novel therapies in this direction makes the cuprizone model a useful tool.

\section{Theiler's murine encephalomyelitis virus}

Viral infections have been hypothesized to be directly or indirectly implicated in the initiation of multiple sclerosis (56). The TMEV infection method was developed by Theiler in $1934(57,58)$ and later established as a model of multiple sclerosis by Lipton (59). This model is induced only in mice. When compared to TMEV, the rat TEV is not as highly virulent. With the exception of evidence published in 2005, rats do not seem to develop brain demyelination (60). In mice, susceptibility to TMEV is modulated by genetic factors. Several susceptibility polymorphic loci have been identified in the mouse genome within the major histocompatibility complex genes and the gene that codes for the beta-chain of the T-cell receptor. These loci modulate the severity of TMEV infection and the length of viral persistence in the brain $(61,62)$.

In mice, the pathology is induced via an intracerebral injection of Picornaviridae, which is a family of single-stranded RNA viruses belonging to the Cardiovirus genus. Two main types of TMEV are known, one highly aggressive that causes an extremely severe neuropathology leading to death within 1 week (induced by GDVII and FA strains of TV), and the other, less aggressive and not fatal (induced by DA and BeAn strains) (63). The latter can induce either a monophasic or a biphasic disease, depending on the mouse strain. The monophasic disease is inducible in most of the murine strains, whereas the biphasic form is inducible only in specific susceptible strains (64). The monophasic type and the first phase of the biphasic type are characterized by acute apoptosis of neurons in gray and white matter, appearing 1 week after the injection of the virus. The monophasic disorder clears out within three weeks and the biphasic disease (usually from 1-month post injection) sets the stage for chronic and progressive inflammation, and demyelination begins. This phase is characterized by the activation of glial cells and macrophages, apoptosis of oligodendrocytes, demyelination, and axonal damage, mostly in the spinal cord. The peak demyelination is reached from the third month of virus injection (65). In parallel with the worsening of the pathology, motor disabilities are observed (66). The neurological effects of TMEV seem to be mediated by the activation of T-lymphocytes, such as the CD8 ${ }^{+}$T-cells, rather than by a direct interaction of the virus with the myelin proteins; moreover, the permanence of the virus in the central nervous system seems to depend on the astrocyte activity that supports viral replication (67). In summary, TMEV is useful to reproduce acute or chronic/progressive phases of the disease $(64,68)$. 


\section{From Animal Models to Human Pathology: Critical Issues}

The EAE model is the most widely used model in multiple sclerosis research. This model is particularly useful to test disease-modifying agents with potential immunomodulatory activity; however, out of the hundreds of drugs tested in the EAE model, only a few have been approved for human use. Indeed, some drugs that attenuate EAE pathology in animals, like anti-tumor necrosis factor (TNF) drugs, actually worsen multiple sclerosis symptoms in humans $(20,69)$. Nevertheless, none of the recommended clinical medications for multiple sclerosis comes from pharmacological experimentations on the two other types of animal models. Despite the undeniable utility of EAE model to test novel medications, the consent of scientific community is not unanimous. For example, one of the main criticisms of the EAE model is that it fails to mimic some important features of multiple sclerosis, especially those concerning the immune system activation: EAE is mainly mediated by $\mathrm{CD}^{+} \mathrm{T}$-cells, whereas, in multiple sclerosis, the $\mathrm{CD} 8^{+} \mathrm{T}$-cells play a predominant role (70). To get around this limitation, researchers have developed a $\mathrm{CD}^{+}$T-cell-mediated EAE (71), thus making this model more suitable for the study of CD8-mediated pathology. In addition, EAE is usually characterized by spinal cord demyelination, and in contrast to human pathology, cortical lesions are nearly absent. Cortical demyelination is a prominent marker of chronic multiple sclerosis. This major limitation can actually be overcome by stereotaxic injection of the MOG directly into the rat cerebral cortex (72). Another critical point is the enormous variability of EAE pathology, due to the different activities of the available antigenic peptides, and to the variable immune responses by the different animal species and strains. For these reasons, the choice of the peptide and of the animal species/strain is critical for study design and data interpretation.

Cuprizone, although it efficiently and consistently reproduces the demyelination and remyelination processes, it cannot be interpreted as an actual model of multiple sclerosis. Nevertheless, it can be used to investigate the molecular mechanisms implicated in oligodendrocyte degeneration and remyelination, in order to identify biological markers for the development of new pharmacological treatments to protect mature oligodendrocytes and to prompt oligodendrocyte precursor maturation.

In contrast to the other two models, the TMEV can be considered an actual model of the pathogenic mechanisms of multiple sclerosis, as the virus infection probably plays a role in the onset of the human disease. In general, when translating from animal models to the human pathology, it is relevant to take into account and investigate why some animals, within the same experimental group, neither develop the disease nor respond to therapies. Most literature does not present negative data, and exclude the "nonresponder" animals from the statistical analysis as outliers. The number of "nonresponders" should also be reported and the origin of this usual variability investigated, as it might be helpful in understanding the human variability with respect to susceptibility to multiple sclerosis, the clinical course, the severity of the disease, and the response to treatment (73). 


\section{Conclusion}

Taking into account the intrinsic limitations of each animal model, we can summarize that the EAE model is mostly relevant for relapsing-remitting multiple sclerosis, which affects the majority of patients (about 80\%).The EAE model is extremely versatile and can be designed to mimic acute and chronic disease courses. The cuprizone intoxication model, although less flexible than the EAE model, is mostly relevant to the acute and chronic courses of disease, but it can be manipulated also to recreate a relapsing-remitting pathology. The TMEV infection is the reference model to study viral-mediated mechanisms of acute and primary progressive multiple sclerosis. Finally, data on animals that do not respond to the disease induction, or treatment, are also essential to explain the variability usually observed in multiple sclerosis patients.

Conflict of interest: The authors declare no potential conflicts of interest with respect to research, authorship, and/or publication of this article.

Copyright and permission statement: To the best of our knowledge, the materials included in this chapter do not violate copyright laws. All original sources have been appropriately acknowledged and/or referenced. Where relevant, appropriate permissions have been obtained from the original copyright holder(s).

\section{References}

1. Lublin FD. The diagnosis of multiple sclerosis. Curr Opin Neurol. 2002 Jun;15(3):253-256. http:// dx.doi.org/10.1097/00019052-200206000-00005

2. Lucchinetti CF, Bruck W, Rodriguez M, Lassmann H. Distinct patterns of multiple sclerosis pathology indicates heterogeneity on pathogenesis. Brain Pathol. 1996 Jul 1;6(3):259-274. http://dx.doi. org/10.1111/j.1750-3639.1996.tb00854.x

3. Lucchinetti C, Brück W, Parisi J, Scheithauer B, Rodriguez M, Lassmann H. Heterogeneity of multiple sclerosis lesions: Implications for the pathogenesis of demyelination. Ann Neurol. 2000 Jun;47(6): 707-717. http://dx.doi.org/10.1002/1531-8249(200006)47:6\%3C707::AID-ANA3\%3E3.0.CO;2-Q

4. Napier MD, Poole C, Satten GA, Ashley-Koch A, Marrie RA, Williamson DM. Heavy metals, organic solvents, and multiple sclerosis: An exploratory look at gene-environment interactions. Arch Environ Occup Health. 2016 Aug 19;71(1):26-34. http://dx.doi.org/10.1080/19338244.2014.937381

5. Bogie JF, Stinissen P, Hendriks JJ. Macrophage subsets and microglia in multiple sclerosis. Acta Neuropathol. 2014 Jun 22;128(2):191-213. http://dx.doi.org/10.1007/s00401-014-1310-2

6. Macchi B, Marino-Merlo F, Nocentini U, Pisani V, Cuzzocrea S, Grelli S, et al. Role of inflammation and apoptosis in multiple sclerosis: Comparative analysis between the periphery and the central nervous system. J Neuroimmunol. 2015 Aug 29;287:80-87. http://dx.doi.org/10.1016/j.jneuroim .2015.08.016

7. Lubetzki C, Stank B. Demyelination in multiple sclerosis. Handb Clin Neurol. 2014;122(chapter 4): 89-99.

8. Höflich KM, Beyer C, Clarner T, Schmitz C, Nyamoya S, Kipp M, et al. Acute axonal damage in three different murine models of multiple sclerosis: A comparative approach. Brain Res. 2013 Sep 1; 1650:125-133. http://dx.doi.org/10.1016/j.brainres.2016.08.048 
9. Wolf A, Kabat EA, Bezer AE. The pathology of acute disseminated encephalomyelitis produced experimentally in the rhesus monkey and its resemblance to human demyelinating disease. J Neuropathol Exp Neurol. 1947 Oct;6(4):333-357. http://dx.doi.org/10.1097/00005072-1947 10000-00003

10. Delarasse C, Smith P, Baker D, Amor S. Novel pathogenic epitopes of myelin oligodendrocyte glycoprotein induce experimental autoimmune encephalomyelitis in C57BL/6 mice. Immunology. 2013 Dec;140(4):456-464. http://dx.doi.org/10.1111/imm.12155

11. Zamora A, Matejuk A, Silverman M, Vandenbark AA, Offner H. Inhibitory effects of incomplete Freund's adjuvant on experimental autoimmune encephalomyelitis. Autoimmunity. 2002 Feb;35(1):21-28. http://dx.doi.org/10.1080/08916930290005873

12. Stosic-Grujicic S, Ramic Z, Bumbasirevic V, Harhaji L, Mostarica-Stojkovic M. Induction of experimental autoimmune encephalomyelitis in Dark Agouti rats without adjuvant. Clin Exp Immunol. 2004 Apr;136(1):49-55. http://dx.doi.org/10.1111/j.1365-2249.2004.02418.x

13. Sun D, Whitaker JN, Huang Z, Liu D, Coleclough C, Wekerle H, et al. Myelin antigen-specific CD8+ $\mathrm{T}$ cells are encephalitogenic and produce severe disease in C57BL/6 mice. J Immunol. 2001;166: 7579-7587. http://dx.doi.org/10.4049/jimmunol.166.12.7579

14. Bettelli E, Korn T, Oukka M, Kuchroo VK. Induction and effector functions of T(H)17 cells. Nature. 2008 Jun;453(12):1051-1057. http://dx.doi.org/10.1038/nature07036

15. Huseby ES, Liggitt D, Brabb T, Schnabel B, Ohlén C, Goverman J. A pathogenic role for myelinspecific CD8(+) T cells in a model for multiple sclerosis. J Exp Med. 2001 Sep;194(5):669-676. http://dx.doi.org/10.1084/jem.194.5.669

16. Patel J, Balabanov R. Molecular mechanisms of oligodendrocyte injury in multiple sclerosis and experimental autoimmune encephalomyelitis. Int J Mol Sci. 2012 Aug 23;13(8):10647-10659. http:// dx.doi.org/10.3390/ijms130810647

17. Lublin FD, Maurer PH, Berry RG, Tippett D. Delayed, relapsing experimental allergic encephalomyelitis in mice. J Immunol. 1981 Mar;126(3):819-822.

18. Yamasaki R, Lu H, Butovsky O, Ohno N, Rietsch AM, Cialic R, et al. Differential roles of microglia and monocytes in the inflamed central nervous system. J Exp Med. 2014 Jul 7;211(8):1533-1549. http:// dx.doi.org/10.1084/jem.20132477

19. Ayers MM, Hazelwood LJ, Catmull DV, Wang D, McKormack Q, Bernard CC, et al. Early glial responses in murine models of multiple sclerosis. Neurochem Int. 2004;45(2/3):409-419. http:// dx.doi.org/10.1016/j.neuint.2003.08.018

20. Constantinescu CS, Farooqi N, O'Brien K, Gran B. Experimental autoimmune encephalomyelitis (EAE) as a model for multiple sclerosis (MS). Br J Pharmacol. 2011 Mar 5;164(4):1079-1106. http:// dx.doi.org/10.1111/j.1476-5381.2011.01302.x

21. Plant GT. Optic neuritis and multiple sclerosis. Curr Opin Neurol. 2008 Feb;21(1):16-21. http:// dx.doi.org/10.1097/WCO.0b013e3282f419ca

22. Bernard CC, Johns TG, Slavin A, Ichikawa M, Ewing C, Liu J, et al. Myelin oligodendrocyte glycoprotein: A novel candidate autoantigen in multiple sclerosis. J Mol Med (Berl). 1997 Feb;75(2):77-88. http://dx.doi.org/10.1007/s001090050092

23. Ichikawa M, Johns TG, Liu J, Bernard CC. Analysis of the fine B cell specificity during the chronic/ relapsing course of a multiple sclerosis-like disease in Lewis rats injected with the encephalitogenic myelin oligodendrocyte glycoprotein peptide 35-55. J Immunol. 1996 Jul;157(2):919-926.

24. Miljkovic D, Stosic-Grujicic S, Markovic M, Momcilovic M, Ramic Z, Maksimovic-Ivanic D, et al. Strain difference in susceptibility to experimental autoimmune encephalomyelitis between Albino Oxford and Dark Agouti rats correlates with disparity in production of IL-17, but not nitric oxide. J Neurosci Res. 2006 Aug 1;84(2):379-388. http://dx.doi.org/10.1002/jnr.20883

25. Tse HY, Li J, Zhao X, Chen F, Ho PP, Shaw MK. Lessons learned from studies of natural resistance in murine experimental autoimmune encephalomyelitis. Curr Trends Immunol. 2012;13:1-12.

26. Kerlero de Rosbo N, Mendel I, Ben-Nun A. Chronic relapsing experimental autoimmune encephalomyelitis with a delayed onset and an atypical clinical course, induced in PL/J mice by myelin oligodendrocyte glycoprotein (MOG)-derived peptide: Preliminary analysis of MOG T cell epitopes. Eur J Immunol. 1995 Apr;25(4):985-993. http://dx.doi.org/10.1002/eji.1830250419 
27. Pöllinger B, Krishnamoorthy G, Berer K, Lassmann H, Bösl MR, Dunn R, et al. Spontaneous relapsingremitting EAE in the SJL/J mouse: MOG-reactive transgenic T cells recruit endogenous MOG-specific B cells. J Exp Med. 2009 Jun 1;206(6):1303-1316. http://dx.doi.org/10.1084/jem.20090299

28. Papenfuss TL, Rogers CJ, Gienapp I, Yurrita M, McClain M, Damico N, et al. Sex differences in experimental autoimmune encephalomyelitis in multiple murine strains. J Neuroimmunol. 2004 May;150(1/2):59-69. http://dx.doi.org/10.1016/j.jneuroim.2004.01.018

29. Croxford AL, Kurschus FC, Waisman A. Mouse models for multiple sclerosis: Historical facts and future implications. Biochim Biophys Acta. 2011 Feb;1812(2):177-183. http://dx.doi.org/10.1016/j. bbadis.2010.06.010

30. Mannie M, Swanborg RH, Stepaniak JA. Experimental autoimmune encephalomyelitis in the rat. Curr Protoc Immunol. 2009 Apr;Chapter 15:Unit 15.2. http://dx.doi.org/10.1002/0471142735. im1502s 85

31. Rivers TM, Schwentker FF. Encephalomyelitis accompanied by myelin destruction experimentally produced in monkeys. J Exp Med. 1935 Apr;61(5):689-702. http://dx.doi.org/10.1084/jem.61. 5.689

32. Revina ES, Gromova NV, Timoshina TE. Changes in phospholipid composition of the spinal cord in rabbits with allergic encephalomyelitis as an experimental model of multiple sclerosis. Bull Exp Biol Med. 2011 Dec;152(2):224-227. http://dx.doi.org/10.1007/s10517-011-1494-6

33. Driscoll EF, Kira J, Kies MW, Alvord EC. Mechanism of demyelination in the guinea pig. Separate sensitization with encephalitogenic myelin basic protein and nonencephalitogenic brain components. Neurochem Pathol. 1986 Feb;4(1):11-22. http://dx.doi.org/10.1007/BF02834295

34. Michalets E, Creger J, Shillinglaw W. Outcomes of expanded use of clinical pharmacist practitioners in addition to team-based care in a community health system intensive care unit. Am J Health Syst Pharm. 2015 Jan;72(1):47-53. http://dx.doi.org/10.2146/ajhpl40105

35. Cross AH, Naismith RT. Established and novel disease-modifying treatments in multiple sclerosis. J Intern Med. 2014 Mar 11;275(4):350-363. http://dx.doi.org/10.1111/joim.12203

36. Rodriguez M. Effectors of demyelination and remyelination in the CNS: Implications for multiple sclerosis. Brain Pathol. 2007 Apr;17(2):219-229. http://dx.doi.org/10.1111/j.1750-3639.2007.00065.x

37. Carlton WW. Response of mice to the chelating agents sodium diethyldithiocarbamate, alpha-benzoinoxime, and biscyclohexanone oxaldihydrazone. Toxicol Appl Pharmacol. 1966 May;8(3):512-521. http://dx.doi.org/10.1016/0041-008X(66)90062-7

38. Praet J, Guglielmetti C, Berneman Z, Van der Linden A, Ponsaerts P. Cellular and molecular neuropathology of the cuprizone mouse model: Clinical relevance for multiple sclerosis. Neurosci Biobehav Rev. 2004 Nov;47:485-505. http://dx.doi.org/10.1016/j.neubiorev.2014.10.004

39. Basoglu H, Boylu NT, Kose H. Cuprizone-induced demyelination in Wistar rats; electrophysiological and histological assessment. Eur Rev Med Pharmacol Sci. 2013 Oct;17(20):2711-2717.

40. Love S. Cuprizone neurotoxicity in the rat: Morphologic observations. J Neurol Sci. 1988 Apr;84 (2-3):223-237. http://dx.doi.org/10.1016/0022-510X(88)90127-X

41. Silvestroff L, Bartucci S, Pasquini J, Franco P. Cuprizone-induced demyelination in the rat cerebral cortex and thyroid hormone effects on cortical remyelination. Exp Neurol. 2012 May;235(1): 357-367. http://dx.doi.org/10.1016/j.expneurol.2012.02.018

42. Oakden W, Bock NA, Al-Ebraheem A, Farquharson MJ, Stanisz GJ. Early regional cuprizone-induced demyelination in a rat model revealed with MRI. NMR Biomed. 2017 May 22; 30(9). [Epub ahead of print]. http://dx.doi.org/10.1002/nbm.3743

43. Hiremath MM, Saito Y, Knapp GW, Ting JP, Suzuki K, Matsushima GK. Microglial/macrophage accumulation during cuprizone-induced demyelination in C57BL/6 mice. J Neuroimmunol. 1998 Jan 23;92(1/2):38-49. http://dx.doi.org/10.1016/S0165-5728(98)00168-4

44. Blakemore WF. Demyelination of the superior cerebellar peduncle in the mouse induced by cuprizone. J Neurol Sci. 1973 Sep 1;20(1):63-72. http://dx.doi.org/10.1016/0022-510X(73)90118-4

45. Ludwin SK. Central nervous system demyelination and remyelination in the mouse: An ultrastructural study of cuprizone toxicity. Lab Invest. 1978 Dec;39(6):597-612.

46. Herder V, Hansmann F, Stangel M, Skripuletz T, Baumgärtner W, Beineke A. Lack of cuprizoneinduced demyelination in the murine spinal cord despite oligodendroglial alterations substantiates the 
concept of site-specific susceptibilities of the central nervous system. Neuropathol Appl Neurobiol. 2011 Oct;37(6):676-684. http://dx.doi.org/10.1111/j.1365-2990.2011.01168.x

47. Palumbo S, Bosetti F. Alterations of brain eicosanoid synthetic pathway in multiple sclerosis and in animal models of demyelination: Role of cyclooxygenase-2. Prostaglandins Leukot Essent Fatty Acids. 2013 Sep 16;89(5):273-278. http://dx.doi.org/10.1016/j.plefa.2013.08.008

48. Yoshikawa K, Palumbo S, Toscano CD, Bosetti F. Inhibition of 5-lipoxygenase activity in mice during cuprizone-induced demyelination attenuates neuroinflammation, motor dysfunction and axonal damage. Prostaglandins Leukot Essent Fatty Acids. 2011 May 8;85(1):43-52. http://dx.doi.org/10.1016/j. plefa.2011.04.022

49. Schultz V, van der Meer F, Wrzos C, Scheidt U, Bahn E, Stadelmann C, et al. Acutely damaged axons are remyelinated in multiple sclerosis and experimental models of demyelination. Glia. 2017 Aug;65(8):1350-1360. http://dx.doi.org/10.1002/glia.23167

50. Janssen K, Rickert M, Clarner T, Beyer C, Kipp M. Absence of CCL2 and CCL3 ameliorates Central Nervous System grey matter but not white matter demyelination in the presence of an intact blood-brain barrier. Mol Neurobiol. 2016 Feb 8;53(3):1551-1564. http://dx.doi.org/10.1007/ s12035-015-9113-6

51. Palumbo S, Toscano CD, Parente L, Weigert R, Bosetti F. The cyclooxygenase-2 pathway via the PGE(2) EP2 receptor contributes to oligodendrocytes apoptosis in cuprizone-induced demyelination. J Neurochem. 2011 Jun 28;121(3):418-417. http://dx.doi.org/10.1111/j.1471-4159.2011.07363.x

52. Bakker DA, Ludwin SK. Blood-brain barrier permeability during Cuprizone-induced demyelination. Implications for the pathogenesis of immune-mediated demyelinating diseases. J Neurol Sci. 1987 Apr;78(2):125-137. http://dx.doi.org/10.1016/0022-510X(87)90055-4

53. Kondo A, Nakano T, Suzuki K. Blood-brain barrier permeability to horseradish peroxidase in twitcher and cuprizone-intoxicated mice. Brain Res. 1987 Nov;425(1):186-190. http://dx.doi. org/10.1016/0006-8993(87)90499-9

54. Ludwin SK. Chronic demyelination inhibits remyelination in the central nervous system. An analysis of contributing factors. Lab Invest. 1980 Oct;43(4):382-387.

55. Johnson ES, Ludwin SK. The demonstration of recurrent demyelination and remyelination of axons in the central nervous system. Acta Neuropathol. 1981;53(2):93-98. http://dx.doi.org/10.1007/ BF00689988

56. Geginat J, Paroni M, Pagani M, Galimberti D, De Francesco R, Scarpini E, et al. The enigmatic role of viruses in multiple sclerosis: Molecular mimicry or disturbed immune surveillance? Trends Immunol. 2017 Jul;38(7):498-512. http://dx.doi.org/10.1016/j.it.2017.04.006

57. Theiler M. Spontaneous encephalomyelitis of mice: A new virus disease. Science. 1934 Aug;80(2066):122. http://dx.doi.org/10.1126/science.80.2066.122-a

58. Mentis AA, Dardiotis E, Grigoriadis N, Petinaki E, Hadjigeorgiou GH. Viruses and endogenous retroviruses in multiple sclerosis: From correlation to causation. Acta Neurol Scand. 2017 May 23. http:// dx.doi.org/10.1111/ane.12775

59. Lipton HL. Theiler's virus infection in mice: An unusual biphasic disease process leading to demyelination. Infect Immun. 1975 May;11(5):1147-1155.

60. Rodrigues DM, Martins SS, Gilioli R, Guaraldo AM, Gatti MS. Theiler's murine encephalomyelitis virus in nonbarrier rat colonies. Comp Med. 2005 Oct;55(5):459-464.

61. Melvold RW, Jokinen DM, Knobler RL, Lipton HL. Variations in genetic control of susceptibility to Theiler's murine encephalomyelitis virus (TMEV)-induced demyelinating disease. I. Differences between susceptible SJL/J and resistant BALB/c strains map near the T cell beta-chain constant gene on chromosome 6. J Immunol. 1987 Mar 1;138(5):1429-1433.

62. Oleszak EL, Chang JR, Friedman H, Katsetos CD, Platsoucas CD. Theiler's virus infection: A model for multiple sclerosis. Clin Microbiol Rev. 2004 Jan;17(1):174-207. http://dx.doi.org/10.1128/ CMR.17.1.174-207.2004

63. Zoecklein LJ, Pavelko KD, Gamez J, Papke L, McGavern DB, Ure DR, et al. Direct comparison of demyelinating disease induced by the Daniel's strain and BeAn strain of Theiler's murine encephalomyelitis virus. Brain Pathol. 2003 Jul;13(3):291-308. http://dx.doi.org/10.1111/j.1750-3639.2003. tb00029.x 
64. Tsunoda I, Fujinami RS. Neuropathogenesis of Theiler's murine encephalomyelitis virus infection, an animal model for multiple sclerosis. J Neuroimmune Pharmacol. 2010 Nov 7;5(3):355-369. http:// dx.doi.org/10.1007/s11481-009-9179-x

65. McGavern DB, Murray PD, Rivera-Quiñones C, Schmelzer JD, Low PA, Rodriguez M. Axonal loss results in spinal cord atrophy, electrophysiological abnormalities and neurological deficits following demyelination in a chronic inflammatory model of multiple sclerosis. Brain. 2000 Mar;123(3):519-31. http://dx.doi.org/10.1093/brain/123.3.519

66. Pirko I, Johnson AJ, Lohrey AK, Chen Y, Ying J. Deep gray matter T2 hypointensity correlates with disability in a murine model of MS. J Neurol Sci. 2009 Jan 21;282(1/2):34-38. http://dx.doi. org/10.1016/j.jns.2008.12.013

67. Zheng L, Calenoff MA, Dal Canto MC. Astrocytes, not microglia, are the main cells responsible for viral persistence in Theiler's murine encephalomyelitis virus infection leading to demyelination. J Neuroimmunol. 2001 Aug;118(2):256-267. http://dx.doi.org/10.1016/S0165-5728(01)00338-1

68. DePaula-Silva AB, Hanak TJ, Libbey JE, Fujinami RS. Theiler's murine encephalomyelitis virus infection of SJL/J and C57BL/6J mice: Models for multiple sclerosis and epilepsy. J Neuroimmunol. 2017 Jul 15;308:30-42. http://dx.doi.org/10.1016/j.jneuroim.2017.02.012

69. The Lenercept Multiple Sclerosis Study Group and The University of British Columbia MS/MRI Analysis Group. TNF neutralization in MS: Results of a randomized, placebo-controlled multicenter study. Neurology. 1999 Aug;53(3):457-465. http://dx.doi.org/10.1212/WNL.53.3.457

70. 't Hart BA, Gran B, Weissert R. EAE: Imperfect but useful models of multiple sclerosis. Trends Mol Med. 2011 Jan 19;17(3):119-125. http://dx.doi.org/10.1016/j.molmed.2010.11.006

71. Steinman L. Myelin-specific CD8 T cells in the pathogenesis of experimental allergic encephalitis and multiple sclerosis. J Exp Med. 2001 Sep;194(5):27-30. http://dx.doi.org/10.1084/jem.194.5.F27

72. Merkler D, Ernsting T, Kerschensteiner M, Brück W, Stadelmann C. A new focal EAE model of cortical demyelination: Multiple sclerosis-like lesions with rapid resolution of inflammation and extensive remyelination. Brain. 2006 May 19;129(8):1972-1983. http://dx.doi.org/10.1093/brain/awll35

73. Patricia JM, Ian SZ. Importance of the non-responder in deciphering animal behavior of experimental autoimmune encephalomyelitis. J Mult Scler. 2016 Jun 15;3:3. 